\title{
NEW STRUCTURE OF THE DRIVE OF SMALL-DIAMETER KNITTING MACHINES
}

\author{
Josef Skrivanek and Martin Bilek
}

Technical University Liberec, Faculty of Mechanical Engineering, Department of Textile and Single-Purpose Machines, Liberec, Czech Republic, josef.skrivanek@tul.cz, martin.bilek@tul.cz

\begin{abstract}
:
This paper is concerned with the drive of small-diameter knitting machines and its optimal structure in reference to the knitting process. Based on an analysis of existing machines, a new structure is proposed employing controlled drives. In this proposal, emphasis has been laid on minimising the consumption of electric power and on simplifying the mechanical section of the structure. An analysis of the dynamic characteristics of the newly proposed concept, and a comparison with existing machines has been carried out.
\end{abstract}

\section{Key words:}

Machine, drive, knitting, structure, design, efficiency.

\section{Introduction}

Circular knitting machines are among the most productive and most employed machines in the knitting industry. From the very beginning circular knitting machines have been developed in two variants; for the manufacture of hosiery products (smalldiameter ones), and for the manufacture of underwear, outerwear and special products (large-diameter ones). Currently, small-diameter knitting machines are overwhelming equipped with one brushless servomotor driving the needle cylinder, the dial and the cutting disk by means of mechanical transmissions. In an effort to make the production as economical as possible, a number of problems arise, related to this dynamic behaviour [1]. These machines are governed by a relatively complicated mechanism with one central drive and a large number of partial transmissions, which reduces the overall efficiency of the output transmission.. The drive systems currently employed do not utilise more efficient individual assemblies, thus resulting in an increased consumption of electric power.

This paper is concerned with an analysis of the existing system of drives of small-diameter knitting machines. The aim is to find a suitable structure that might reduce the energy demand of the knitting process, and this requires the optimisation of the most common system employed; a set of gear wheels that drives the whole system by means of a central drive. The operating elements in this case are; the needle cylinder, the dial, and the cutting disk.

A suitable arrangement of the structure of the drives can also reduce the required installation space in comparison with the present designs. Another important criterion for assessing the suitability of its use is the demand on the dynamics of the machine. The present acceleration of production and minimisation of production times imposes demands on mechanical transmissions, such that they may encounter operational limitations depending on their application.

\section{Present concept of the drive}

The present concepts of the driving system of needle cylinders are based on the principle of central drives and subsequent transmission of their output, provided by means of gear wheels or belts [2].
One of the most often employed concepts of driving systems of knitting machines, described in Figure 1, is based on the transmission of the output on needle cylinder (4) and on the rib dial (10) from the servomotor (M1), by a chain of gear wheels $(1,2,3,5,6,7,8,9)$ and shafts $(11,12,13)$.

The main motion of the knitting machine is realised by means of a special brushless A.C. servomotor (M1), a pinion (1), and toothed ring (2), which form a part of the needle cylinder (4). The drive of the dial is realised by transmission from the toothed ring (2), to the split wheel (3), designed so as to be able to take up plays. Next, it continues by the shaft (11) to the corrected gear set (5-6), manufactured and mounted in such a way that it may be possible to change the axial distance, but also with the purpose of taking up plays. Thus, coincident velocities of revolutions are provided to the dial (10) and the needle cylinder (4). The shaft (12) is equipped with a coupling, which must allow for a repeated precise setting to the original position after tilting of the arm of the dial (10) and the rest of the drive.

During the non-reversible rotation of the cylinder, there are knitted the welt, the leg, the foot and the extra courses. However, for knitting the reverse heel and toe, it is necessary to change to reversible operation, when the machine runs at reduced rpm. When knitting the heel and the toe, the drive of the dial and the cylinder perform a reverse swinging motion $\left(270^{\circ}\right)$ in both senses of the rotation. From a dynamic point of view, the knitting of the reverse heel and toe is considered to be the least favourable state $[3,4]$.

The dial of the knitting machine (the rib dial) is a rotary disk with spreaders, seated in radial grooves. The spreaders are governed by means of fixed cams. The drive of the dial of the knitting machine is realised by a system of gear wheels and shafts, providing not only the dial drive, but also for its proper setting with respect to the movement of needle cylinder. For a proper function of the machine, as well as for an accurate setting of the dial of the knitting machine with respect to the needle cylinder, the following construction conditions for seating the dial must be observed: 1) The seating of the dial must secure its alignment with the needle cylinder, the maximum allowed value of misalignment is $0.05 \mathrm{~mm}, 2$ ) When dismounting and subsequently remounting some parts of the drive of the needle cylinder, or of the dial of the knitting machine, the accurate re-seating of the dial with respect to the needle 


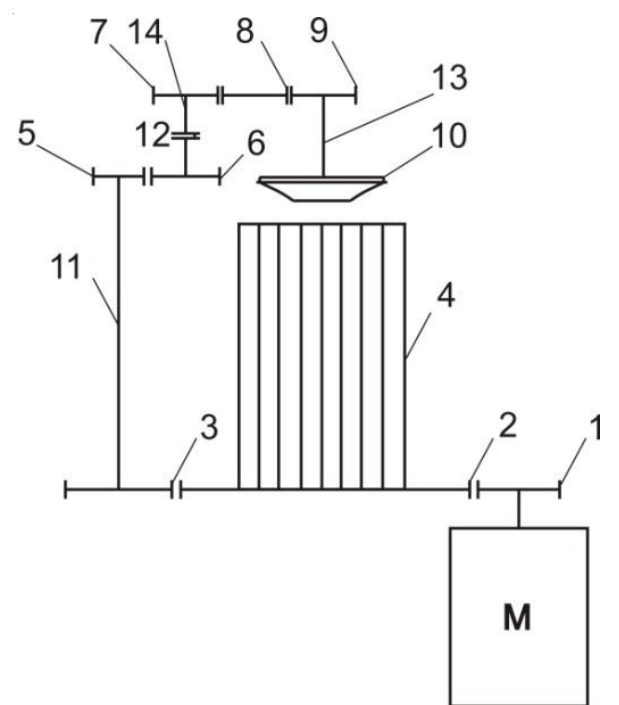

Figure 1. Diagram of the drive of a knitting machine.

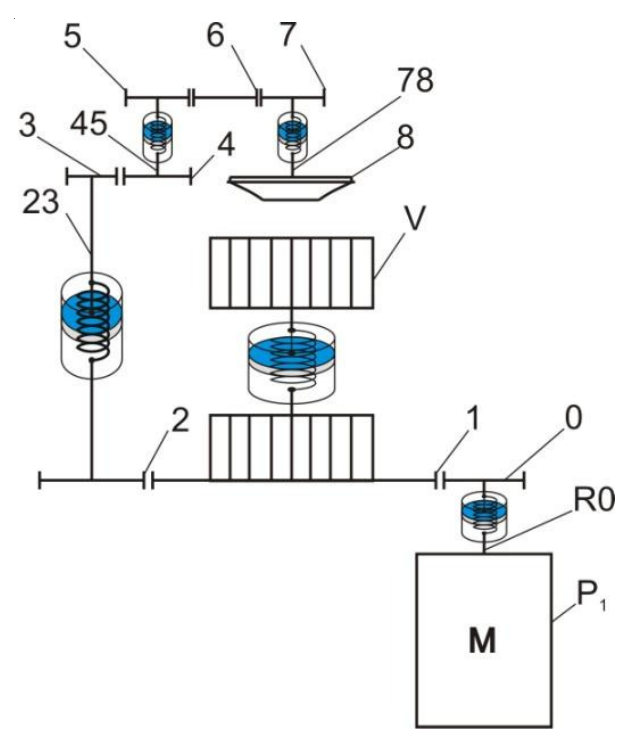

Figure 2. Mathematical model of the drive of cylinder and dial of a knitting machine.

cylinder must be strictly respected, 3) Mutual slewing of the cylinder and the dial of the knitting machine must have a tolerance of \pm 0.05 on a diameter of $100 \mathrm{~mm}$.

\section{Mathematical model of the present concept of the drive}

In order to be able to describe the behaviour of the system under examination, it is necessary to compile a suitable mathematical model that will describe its behaviour during an operation cycle with the maximum possible precision. The individual parts of the system are affected by a number of forces, the magnitudes of which are determined by technological processes and are variable with time, e.g., the forces required for the lift of needles and sinkers, passive resistances in individual kinematic couples, passive resistances in the groove of needle cylinder etc. However, from the point of view of overall loading of partial junctions of the system, the magnitude of some of these forces is negligible, and it is therefore possible to disregard them when compiling the mathematical model without any adverse effect on the accuracy of the solution. Based on these assumptions a substitute diagram of the drive
(Figure 2) was devised. The drive is substituted with a discreet chain $n$, of concentrated masses $I_{1 \ldots n}$, connected by flexible and damping links with the rigidities $k_{1, n}$ and internal damping $b_{1}$. The prescribed course of acceleration of the mechanism is incorporated in the model by means of kinematic excitation of the rotor of the servomotor.

The mathematical model of the driving system of knitting machines (Figure 2) has been devised under the following conditions:

a) the masses of individual elements of the mechanism (elements 0-8) including the corresponding part of shafts and seating are substituted by mass points. With the mass points, their moments of inertia are established.

b) the shafts $(R 0,23,45,78)$ that interconnect the gear wheels transmitting the driving moment are considered to be flexible elements, and their torsional rigidities have been included.

c) the mass of the needle cylinder (9) is considered, including needles, sinkers and its other parts. It is divided into two mass points interconnected by a torsional spring.

d) elastic elements are damped by viscous damping with the damping co-efficient $b$.

e) the calculation considers the plays in kinematic couples and these plays are substituted with angular deflections of the gear wheels of the mechanism.

For compiling the motion equations of the system defined in this way, Lagrange equations of the second kind were employed in the following form:

$$
\frac{\mathrm{d}}{\mathrm{dt}}\left(\frac{\partial \mathrm{E}_{\mathrm{K}}}{\partial \mathrm{q}_{\mathrm{i}}}\right)-\frac{\partial \mathrm{E}_{\mathrm{K}}}{\partial \mathrm{q}_{\mathrm{i}}}+\frac{\partial \mathrm{E}_{\mathrm{P}}}{\partial \mathrm{q}_{\mathrm{i}}}=\mathrm{Q}_{\mathrm{j}}-\frac{\partial \mathrm{R}_{\mathrm{d}}}{\partial \dot{\mathrm{q}}_{\mathrm{i}}}
$$

where: $E_{K}$ - stands for kinetic energy, $E_{P}$ - for potential energy, $R_{d}$ - dissipative function, $q_{i}$ - generalised co-ordinate. For the system shown in Figure 2, the following motion equations can be compiled:

$$
\begin{aligned}
& \ddot{\varphi}_{0}\left(\mathrm{I}_{0}+\mathrm{I}_{1} \eta_{01}^{2}+\mathrm{I}_{2} \eta_{02}^{2}\right)+\mathrm{b}_{0 \mathrm{R}}\left(\dot{\varphi}_{0}-\dot{\varphi}_{\mathrm{R}}\right)-\mathrm{b}_{\mathrm{V}}\left(\dot{\varphi}_{\mathrm{V}}-\dot{\varphi}_{1}\right) \eta_{01}- \\
& \mathrm{b}_{23}\left(\dot{\varphi}_{3}-\dot{\varphi}_{2}\right) \eta_{02}+\mathrm{k}_{0 \mathrm{R}}\left(\varphi_{0}-\varphi_{\mathrm{R}}\right)-\mathrm{k}_{\mathrm{V}}\left(\varphi_{\mathrm{V}}-\varphi_{1}\right) \eta_{01}- \\
& -\mathrm{k}_{23}\left(\varphi_{3}-\varphi_{2}\right)=0
\end{aligned}
$$

$\mathrm{I}_{8} \ddot{\varphi}_{8}+\mathrm{b}_{78}\left(\dot{\varphi}_{8}-\dot{\varphi}_{7}\right)+\mathrm{k}_{78}\left(\varphi_{8}-\varphi_{7}\right)=0$

where $b_{i}$ - is the co-efficient of viscous damping [Nm.s.rad $\left.{ }^{-1}\right], k_{i}$ - co-efficient of rigidity [Nm.rad-1], - transmission, I - moment of inertia [kg. $\left.\mathrm{m}^{2}\right], \phi$ - slewing [rad], $\phi$ - angular velocity [rad. $\left.\mathrm{s}^{-1}\right], \phi$ - angular acceleration [rad. $\mathrm{s}^{-2}$ ]. The inferior letters in designations of variables express their relations to the elements of the drive in Figure 2. 
The main parameters for the solution of the dynamic model are; the moments of inertia, rigidity and damping of the individual elements of the drive, of the cylinder and of the dial of small-diameter knitting machines. All the parts of the driving system have been devised as a 3D model by means of CAD software Pro/Engineer. By using these models the moments of inertia of the elements of the system and torsional rigidities of shafts, of individual trains of gear wheels and of the needle cylinder have been established. Another parameter included in the equations is the co-efficient of viscous damping. The relation for the calculation of these coefficients is based on; the damping logarithmic decrement, moment of inertia, and rigidity of the component. The plays have been incorporated in the solution by means of the following conditions:

$\left|\varphi_{i P}-\varphi_{i}\right| \leq \Phi_{i} \Rightarrow \varphi_{i P}-\varphi_{i}=0$

$\varphi_{i P}-\varphi_{i}>\Phi_{i} \Rightarrow \varphi_{i P}-\varphi_{i} \mapsto \varphi_{i P}-\varphi_{i}-\Phi_{i}$

$\varphi_{i P}-\varphi_{i}<-\Phi_{i} \Rightarrow \varphi_{i P}-\varphi_{i} \mapsto \varphi_{i P}-\varphi_{i}+\Phi_{i}$

where: $\mathrm{i}=0,1,2,3,4,5,6,7,8$.

For the derived motion equations a mathematical model was compiled in MATLAB and for the solution of these equations Matlab, including its superstructure Simulink was used. The individual equations have been written in matrix form and subsequently solved by gradual integration by means of the solver in Simulink. Also used was a solver employing the standard method Dormand-Prince termed ode45.

\section{Results of dynamic analysis of the present concept of the drive}

By solution of the compiled motion equations for the given initial conditions we obtained the response of the system to a stipulated kinematic excitation, namely the course of acceleration when knitting the heel or the toe of a sock, i.e., a swinging motion with a return to the original position. This knitting regime was chosen because it presents the highest demands on the driving system. The course of acceleration is idealised and it is assigned to the rotor of the electric motor.

Figure 3 shows the courses of acceleration of individual elements of the drive with respect to time. By gradual integration in time we obtained the courses of the velocity and of the position. The results of the analysis show an important effect of the plays in the system; changes of direction in the movement of individual gear wheels in the trains bring about large impact forces. It can be seen in Fig. 3, that there are significant peaks of acceleration generated by the existing plays and subsequent mutual collisions of individual teeth. An impact in a gear set generates a temporary oscillating effect, which is then gradually reduced due to viscous damping. These values are as much as three times higher in comparison with the concept of a system without plays.

Similarly, although less markedly, the existing plays also show in the courses of velocity. In certain sections of the courses of velocity there can be seen deviations compared to the theoretical course. The plays in the system and the elasticity of individual elements of the system create an inaccuracy in the position of the output element of the kinematic structure (the dial - element 8) with respect to the input element (knitting cylinder - element V). Figure 4 shows the courses of acceleration of individual elements of the drive and Figure 5 shows the course of differences in the slewing of the dial with respect to the needle cylinder.

\section{New design variants of the drive}

The concepts of the new drive try to maintain a stable mechanical link between the knitting cylinder and the dial. This conceptual direction is based on the case of knitting a sock, where the overall production time amounts to approximately 2 min. The knitting cylinder constantly participates in the knitting process, whereas the cutting disk is involved for a maximum of $50 \%$ of the overall knitting time, and the dial itself (without the saw disk) is used only for a period of several seconds during several revolutions. This is true for the systems that will, be described below. When comparing systems with conventional drives and the optimised drive with separated motions, the optimised version shows an economy of energy of about $30 \%$ [5].

The mechanical arrangement relating to partial drives is drawn in Figure 6, and shows the driving system of a single-cylinder circular knitting machine for manufacturing hosiery goods, which is equipped with two motors; one for the drive of the needle cylinder, and the other to drive the dial with cutting saw. One motor is connected to needle cylinder and the other one to the rib dial. Electrically, both the motors are connected in parallel, and the line to the motor for the drive of the rib dial incorporates a switch governed from the control system of the machine, which brings this motor into operation only for the time when it is needed.

There are known single-cylinder hosiery circular knitting machines where the drive of the rib dial is derived from the needle cylinder, with a coupling incorporated in this drive that serves to connect and disconnect the drive of the rib dial. A disadvantage of this mechanism is the fact that for the control of the coupling it is necessary to reduce the change-over revolutions of the machine. An advantage of the described solution is the fact that it is possible to employ multiple revolutions for the drives of the machine by means of a motor governed by a frequency converter of revolutions, without securing the synchronisation of revolutions of the needle cylinder and of the rib dial mechanically, e.g., by replacement of pulleys.

The equipment arrangement of this invention is shown schematically in Figure 8, outlining the drives of the needle cylinder and of the rib dial. A well-known single-cylinder circular knitting machine of the Ange series for the manufacture of hosiery goods was provided with a needle cylinder (1), driven from electric motor (M1) by mechanical transmission including pulleys (2, 3), and indented belt (4). Above the needle cylinder (1) there is pivoted a rib dial (5) with a cutting saw, and it is driven from electric motor (M2) by mechanical transmission including pulleys $(6,7)$, indented belt (8), shaft (9), pulleys (10, 11 ), belt (12) and shaft (13). However, this patent does not yet introduce the individual natures of the motions of the cutting disk and of the dial yet. Consequently, it is still necessary to drive this dynamically important section of the drive for at least $50 \%$ of the overall knitting time. In parallel, this research is also concerned with the improvement of the properties of the partial elements, investigating the suitability of employing silentblocks that influence the rigidity of the frame, independently of alterations to the frame itself for the purpose of economy.

\section{New structure of the drive of Ange 18.1}

The compiled mathematical model (Figure 8) is intended as a general description of the mechanical section of the system, where certain factors have not been included in the model, 


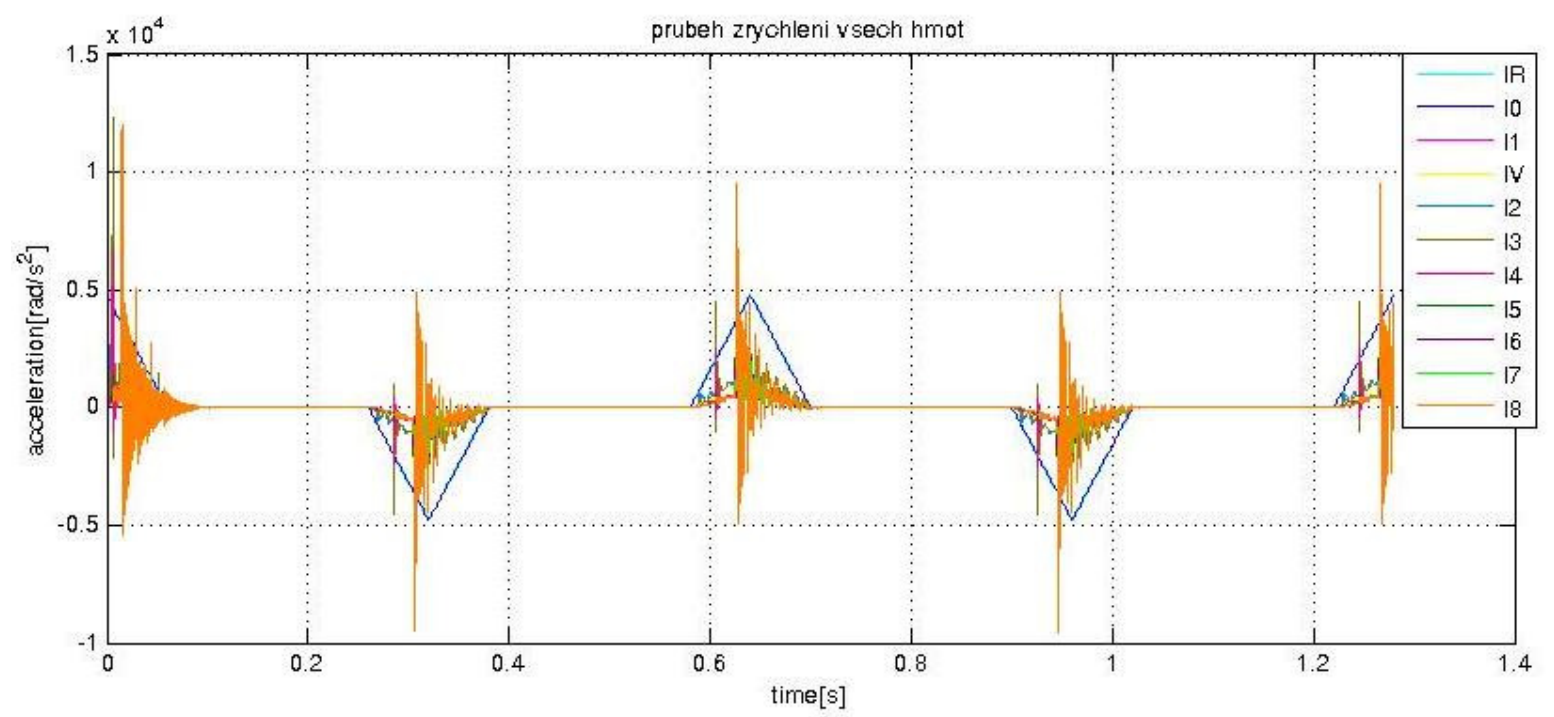

Figure 3. Courses of acceleration of individual elements of the drive.

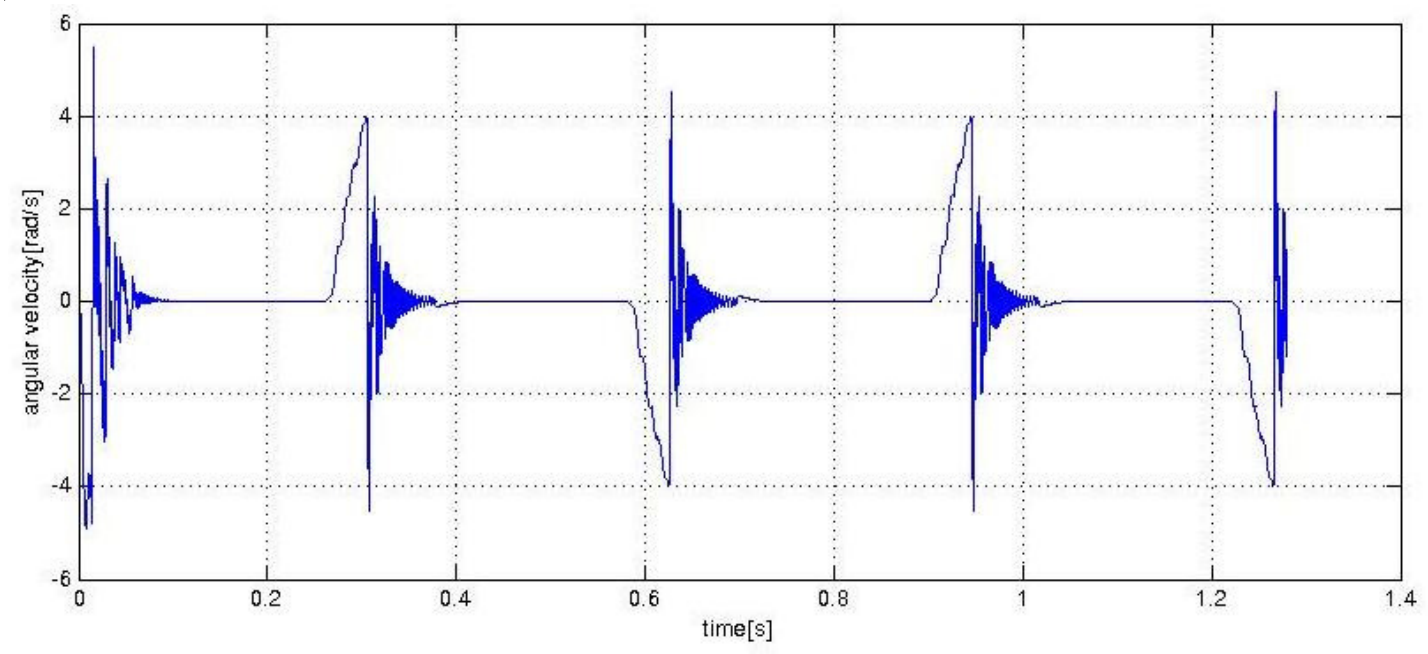

Figure 4. Difference between angular velocities of the dial and the needle cylinder.

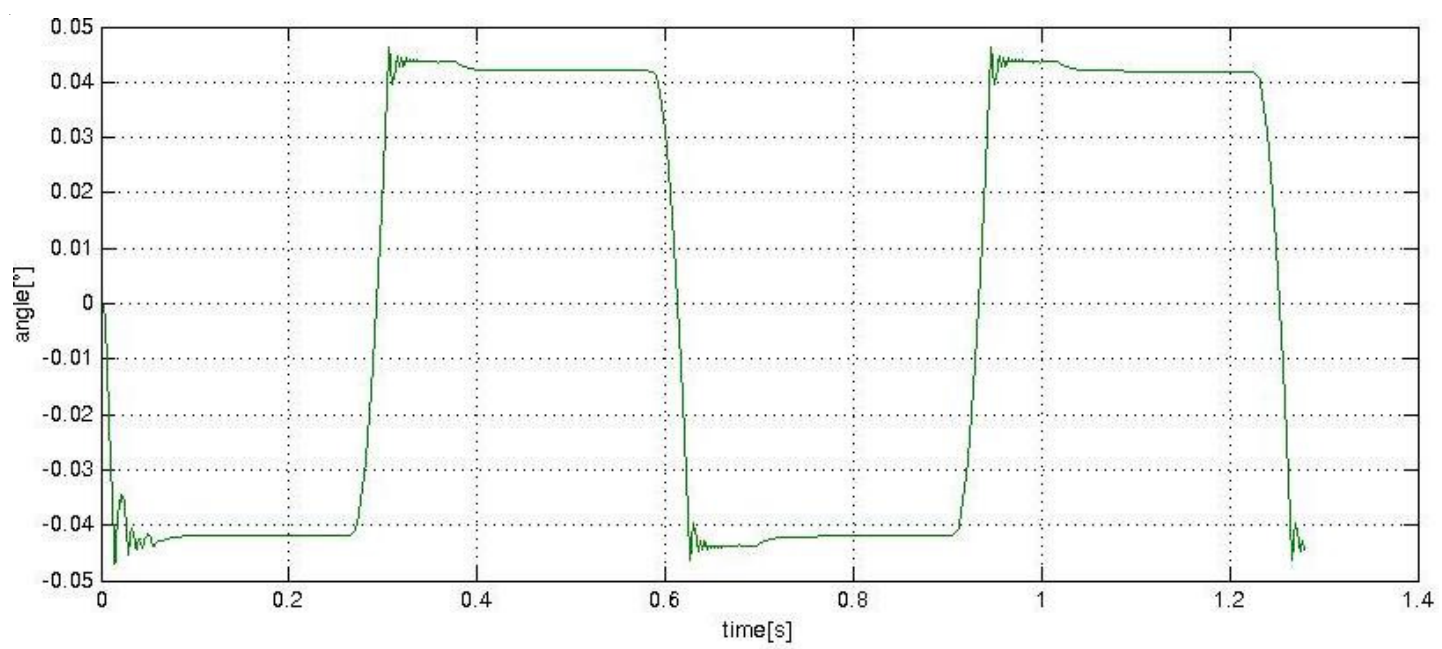

Figure 5. Difference of positions of mutual slewing of the dial and the needle cylinder.

with a partial simplification as a consequence. Two of the factors omitted to simplify the model, are the presence of moving sinkers in the needle grooves, and of needles in the machine cams. Also excluded are the drive of the cutting disk (M3), and the mass of the disk (9), its seating and pulley (10), which are included in the moment of inertia of the dial. On the other hand, the model takes into consideration the effects of play in the kinematic chain. Due to the nature of the task in compiling the motion equations and subsequently, a mathematical model, Lagrange equations of the second kind 


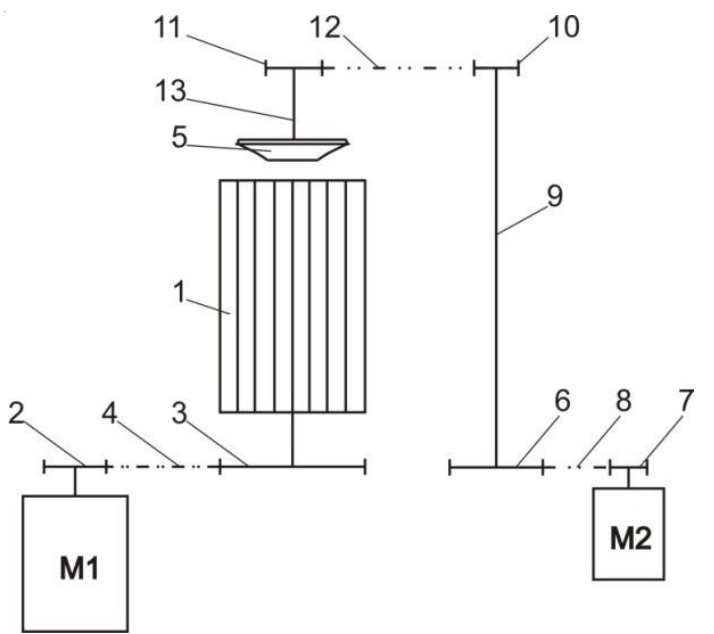

Figure 6. Example of the patented structure.

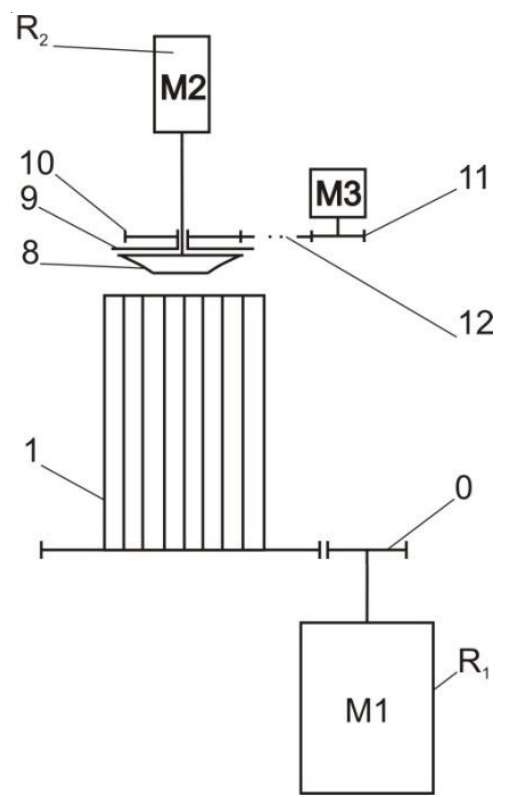

Figure 7. Diagram of new structure of the drive of a knitting machine.

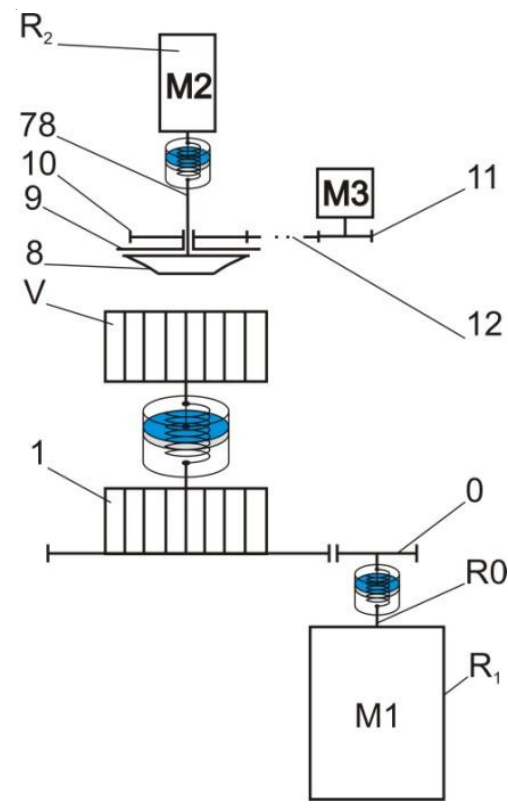

Figure 8. Mathematical model of new structure of the drive. have again been employed. In this present case, these equations are an ideal tool for examination of relations among the motions of the individual elements of the dynamic model, i.e., for describing the mechanical section of the drive.

Through solution of the compiled motion equations for the given initial conditions, we obtained the response of the system, which consisted of an examination of the movement of the chain independent of the prescribed kinematic excitation by the acceleration of the rotor.

The proper mathematical model of the driving system of knitting machines has been generated under identical conditions as that of the original system of the drive of Ange 18.1, and the solver of the differential equations was structured analogously.

Motion equations:

$$
\begin{aligned}
& I_{0} \ddot{\varphi}_{0}+b_{0 R}\left(\dot{\varphi}_{0}-\dot{\varphi}_{R 1}\right)-b_{10} \eta_{01}\left(\dot{\varphi}_{1}-\dot{\varphi}_{0} \eta_{01}\right)+ \\
& +k_{0 R}\left(\varphi_{0}-\varphi_{R 1}\right)++k_{10} \eta_{01}\left(\varphi_{1}-\varphi_{0} \eta_{01}\right)=0 \\
& I_{1} \ddot{\varphi}_{1}+b_{10}\left(\dot{\varphi}_{1}-\dot{\varphi}_{0} \eta_{01}\right)-b_{V}\left(\dot{\varphi}_{V}-\dot{\varphi}_{1}\right)+ \\
& +k_{10}\left(\varphi_{1}-\varphi_{0} \eta_{01}\right)-k_{V}\left(\varphi_{v}-\varphi_{1}\right)=0 \\
& \quad \ddot{\varphi_{v}}+b_{v}\left(\dot{\varphi}_{v}-\dot{\varphi}_{1}\right)+k_{v}\left(\varphi_{v}-\varphi_{1}\right)=0 \\
& I_{8} \varphi_{8}+b_{R 8}\left(\dot{\varphi}_{8}-\dot{\varphi}_{R 2}\right)+k_{R 8}\left(\varphi_{8}-\varphi_{R 2}\right)=0
\end{aligned}
$$

Figures 9 and 10 visualise the most important results of this analysis. It is evident that this system is more advantageous than the current one, regarding the technological condition of mutual slewing of the knitting cylinder with respect to the dial.

\section{Conclusion}

Our analysis of the present situation suggests the development of a concept with three unit drives for the cylinder, dial and cutting disk would bring many benefits in comparison with the systems currently employed.

A reduced power input to the machine would be required, through utilisation of the working diagram of knitting operation for precise adjustment of operations of unit drives, and reduction of the number of mechanical components transmitting the output. Production costs would be reduced due to the suppression of the majority of gear wheels, and thus, a considerable simplification could be achieved not only of the kinematic chain of the drive, but also of the overall structure of the frame of the knitting machine. From the analysis of the optimised drive, a reduction of the angular deflection of mutual slewing of the knitting cylinder and the dial can be seen. There would be a reduction of vibrations and noise. Further development will concern the structure of the seating of the cutting disk, so as not to disrupt technological dependencies; the same will be included in the mathematical model, in order to make the calculation more precise.

Following this will be the definition and programming of the control algorithm based on the working diagram of knitting process. After theoretical dynamic tuning of the system, it will be necessary to manufacture a functional model with three unit drives, in order to verify the suitability of the above-mentioned concepts and this model will incorporate the driving system of the Ange 18.1 optimised drive. Once this functional model has been brought into operation and tuned, there will follow an assessment of its properties. On the basis of data obtained 


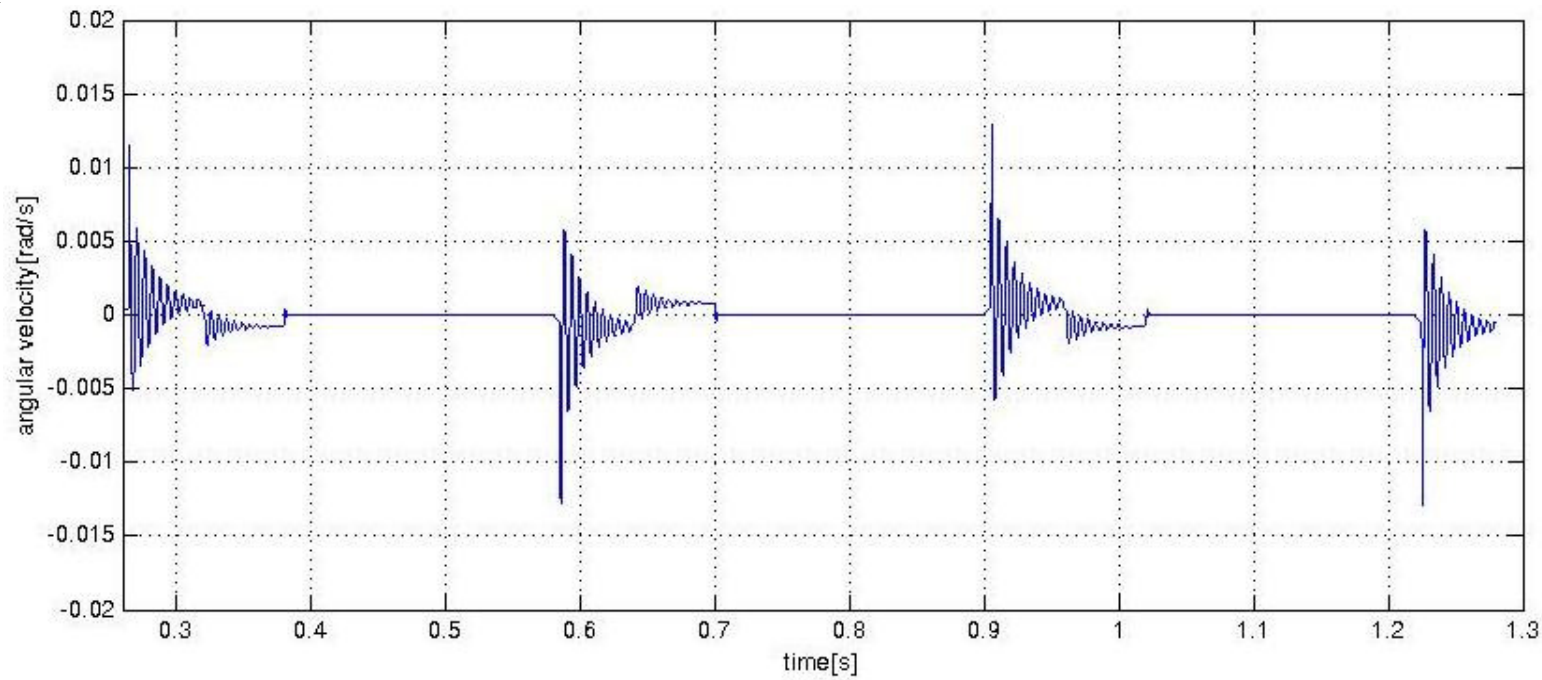

Figure 9. Difference of angular velocities of the dial and of the knitting cylinder.

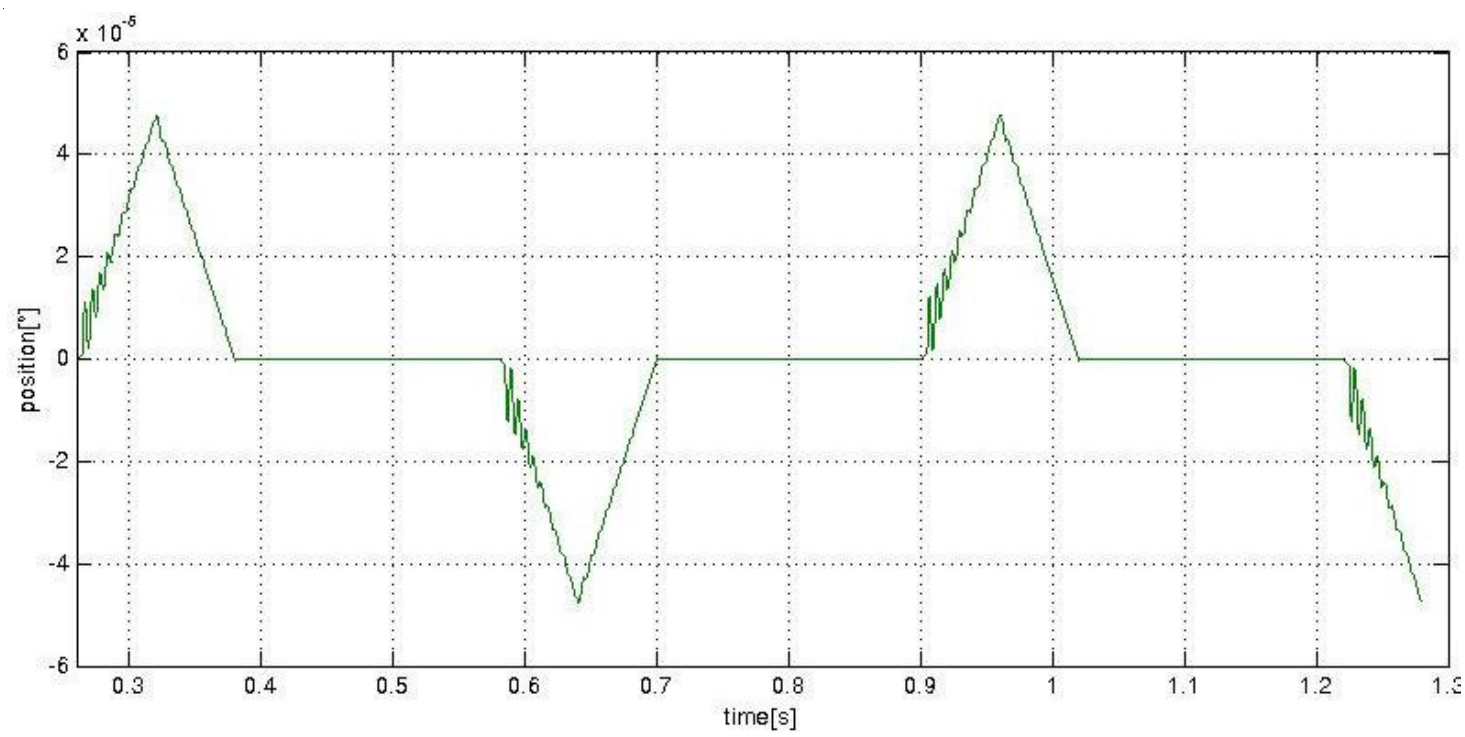

Figure 10. Difference of positions of mutual slewing of the dial and of the knitting cylinder.

during this whole process, an evaluation of the optimised drive should be carried out.

\section{Acknowledgements:}

The paper has been elaborated in the frame of the solution of the grant project: 1 M0553.

\section{References:}

1. Skrivánek, J., Bílek, M.: Analysis of mathematical model of the frame of small- diameter knitting machine, International symposium in knitting and apparel Iska2010, iasi, Romania, 2010, ISSN 2069-1564, 100\%

2. Skrivánek, J., Bílek, M.: Analysis of dynamic model of the drive of small diameter knitting machines Ange 18.1, Acc Journal XVII 2011, Liberec, 2011. ISSN 1803-9782

3. Vaclavik, M., Jirasko, P., "Research and Application of Displacement Laws of Electronic Cams", Proceedings of the 13th IFToMM World Congress, Besancon, France, June 18-21, 2007

4. Mrázek, J.: Theoretical analysis of dynamics four-bar beat up mechanisms of a loom. In.: Mechanism and machine theory, Pergamon Press, 1992, USA
5. Tesar, D., "Intelligent Mechanical Systems", Plenary Paper, Proceedings of the 11th IFTOMM World Congress in Mechanism and Machine Science, Tianjin, China, April 14, 2004. 